\title{
Observatorio de televisión a partir del uso de pantallas en las instituciones educativas Ciudadela Educativa de Bosa IED y el Gimnasio Campestre Reino Británico ${ }^{1}$
}

\author{
Television observatory from the use of screens in educational institutions Educational \\ Ciudadela Educativa de Bosa IED and Gimnasio Campestre Reino Británico
}

\section{Adriana María González Giraldo² \\ Holanda González Valderrama ${ }^{3}$}

\author{
González G, Adriana. González V. Holanda \\ miradas $\mathrm{N}^{\circ} 1-2018$ ISSN digital $\mathrm{N}^{\circ} 2539-3812$ Págs 9 - 27 \\ Recepción: Agosto 22 de 2017 \\ Aprobación: Marzo 15 de 2018 \\ Publicación: junio 29 de 2018
}

\begin{abstract}
"La televisión es el primer sistema verdaderamente democrático, el primero accesible para todo el mundo y completamente gobernado por lo que quiere la gente. Lo terrible es, precisamente, lo que quiere la gente". Clive Barker (1952-?) Escritor y director de cine inglés.
\end{abstract}

\section{Resumen}

Se presentan los hallazgos de la investigación realizada a manera de tesis de la Maestría en Comunicación Educativa de la Universidad Tecnológica de Pereira y la Corporación Universitaria Minuto de Dios. Se toma la televisión como una de las pantallas más utilizadas a través de la cual los contenidos de ficción transmiten sus distintos mensajes, abordando como referente teórico al investigador mexicano Guillermo Orozco. En la práctica desarrollamos algunos de los talleres que este autor propone, e implementamos un Observatorio de televisión en el aula de clases que pretendía observar hasta dónde quienes participaron fortalecen su capacidad para recepcionar la televisión y sus

1 Este artículo es producto de los resultados obtenidos en el Trabajo de grado presentado como requisito para optar al título de Magister en Comunicación Educativa, “OBSERVATORIO DE TELEVISIÓN A PARTIR DEL USO DE PANTALLAS EN LAS INSTITUCIONES EDUCATIVAS CIUDADELA EDUCATIVA DE BOSA IED Y EL GIMNASIO CAMPESTRE REINO BRITÁNICO”UNIVERSIDAD TECNOLÓGICA DE PEREIRA EN CONVENIO CON LA CORPORACIÓN UNIVERSITARIA MINUTO DE DIOS (UNIMINUTO), en Mayo de 2016.

2 Licenciada en español y literatura de la U. Tecnológica de Pereira, especialista en comunicación educativa de la Corporación Universitaria Minuto de Dios, y magíster en Comunicación Educativa de la Universidad Tecnológica de Pereira en convenio con la Corporación Universitaria Minuto de Dios, experiencia de 9 años como docente en primaria, básica secundaria y media vocacional, actualmente se desempeña como docente en el Colegio Abraham Lincoln. adrianamaria_454@hotmail.com

3 Licenciada en Pedagogía Infantil de la Corporación Universitaria Iberoamericana, Especialista en Comunicación Educativa de la Corporación Universitaria Minuto de Dios y Magister en Comunicación Educativa de la Universidad Tecnológica de Pereira en convenio con la Corporación Universitaria Minuto de Dios, experiencia de 8 años como docente de Primera Infancia y 7 años en Básica Primaria, actualmente docente en el Colegio Ciudadela Educativa de Bosa, IED. hogova59@gmail.com 
contenidos de ficción de manera crítica, en calidad de audiencias, identificando las micro y macro-mediaciones, como eje fundamental del objetivo general propuesto para la investigación. Así mismo, se buscó determinar las concurrencias en las distintas producciones realizadas por los estudiantes.

La muestra corresponde a 28 estudiantes, niños y jóvenes, en edades entre los 9 y 18 años. Como instrumento para la recolección de datos se utilizó una encuesta, cuyo modelo fue adoptado de aquel propuesto por la Universidad Tecnológica de Pereira -al igual que el análisis de los datos obtenidos-También recurrimos a la observación participante adaptada y a los diarios de campo para el análisis de cada actividad y las producciones obtenidas.

En las conclusiones se da respuesta a la pregunta de investigación, y se muestran las similitudes y diferencias logradas en las producciones desarrolladas por los dos grupos de estudiantes participantes del observatorio de televisión de las dos instituciones educativas.

Palabras claves: televisión, pantallas, observatorio de televisión, audiencias, micromediaciones, macromediaciones.

\section{Abstract}

The current research findings were carried out as a thesis for the Masters in Educative Communication of the Universidad Tecnológica de Pereira and the Corporación Universitaria Minuto de Dios.

In this investigation, Television is understood as one of the most used screens where fiction contents show a variety of messages, addressing as a theoretical reference the Mexican researcher: Guillermo Orozco. In practice here were developed some of the workshops that this author proposes, likewise it was implemented a Television Observatory in the classroom, which sought to observe how the participants of this analysis strengthen their capacity to receive television and its contents of fiction in a critical way, as audiences, identifying the micro and macro-mediations, as a fundamental axis of the general objective proposed for this research. Furthermore, it points out to determine the concurrences in the different productions made by the students.

The sample corresponds with 28 students, children, and young people, between the ages of 9 and 18 years. A survey was used as an instrument for data collection, whose model was adopted from that proposed by the Universidad Tecnológica de Pereira as well as the analysis of the data obtained. We also resort to the adapted participant observation and the field journals for the analysis of each activity and the obtained productions.

The conclusions answer the research question; these also show the similarities and differences achieved in the productions developed by the two groups of students participating in the television observatory of the two educational institutions.

keywords: screens, television, contents of fiction, audiences, identifying the micro and macro-mediations.

\section{Introducción}

El presente artículo busca mostrar el trabajo y resultados obtenidos en la investigación titulada Observatorio de televisión a partir del uso de pantallas 
en las instituciones educativas Ciudadela Educativa de Bosa IED y el Gimnasio Campestre Reino Británico, el cual se trabajó desde el modelo de investigación cualitativa, permitiendo afirmar que el uso de la televisión y de otras pantallas que ofrecen las nuevas tecnologías de la información y comunicación, brindan la posibilidad de acceder a una gran variedad de contenidos ofertados por las diferentes programadoras y la internet, siendo la televisión la más utilizada, ya que a través de esta, las audiencias infantil y juvenil reciben infinidad de mensajes que pueden ser adecuados o no, que influyen en las concepciones o formas de ver y entender la realidad, asociándola directamente con su entorno. Es precisamente este aspecto lo que nos generó un cuestionamiento respecto a la forma en que los niños y jóvenes están asumiendo la televisión, sus contenidos, mensajes y cuál es esa relación con el contexto educativo.

A través de los contenidos televisivos se puede evidenciar según la franja y horario, la reglamentación, el tipo de público al que van dirigidos los contenidos a los que se puede acceder, sin embargo, esto no es suficiente y es necesario que mediaciones como la de la familia sea inmediata, al igual que la representada por la escuela como coadyuvante en ese proceso de mediación, con el fin de orientar la forma en que las audiencias infantil y juvenil recepcionan los contenidos televisivos, de tal forma que se puedan generar espacios de interacción crítica.

La televisión tiene la posibilidad de ofrecer diferentes alternativas $\mathrm{u}$ opciones en sus contenidos que pueden generar aprendizajes; sin embargo este aspecto sigue siendo un tema de discusión en el entorno educativo, es por esta razón que el proyecto de investigación lo orientamos a la creación de un observatorio de televisión, analizando su desarrollo en el aula de clase, para este, planteamos el problema desde el siguiente interrogante: ¿Es posible distinguir las micro y macro mediaciones en la recepción de contenidos televisivos de ficción con los estudiantes de grado quinto de la Institución Educativa Distrital Ciudadela Educativa de Bosa y los estudiantes de grado undécimo del colegio Gimnasio Campestre Reino Británico, a través de la creación de un observatorio de televisión?.

Para el desarrollo del observatorio de televisión tomamos como referente teórico principal al investigador mexicano Guillermo Orozco, también nos apoyamos en otros autores como Carlos Scolari y Jesús Martín-Barbero.

Al acercarnos a los planteamientos de Guillermo Orozco y Mercedes Charles, es pertinente hablar de los procesos de significación que hace el niño frente a los mensajes televisivos y el rol que ejercen las instituciones sociales como mediadoras, como lo expresan los autores en el texto "Hacia una lectura crítica de los medios", en el que manifiestan que "[...] las significaciones propuestas por la escuela y la familia son diferentes, y a veces no se complementan, por lo menos tienden a no contradecirse. En cambio, las diferencias en la significación de guiones entre estas dos instituciones y la televisión tienden a representar competencia y conflicto." (Orozco \& Charles, 1987), partiendo de esta afirmación volvemos a la concepción de que los niños y jóvenes requieren de una orientación clara y de espacios de interacción para interiorizar, apropiar, 
distinguir qué es pertinente o no para su vida a partir de lo que emite la TV.

Para desarrollar el proyecto en las dos instituciones educativas distantes una de la otra y con poblaciones distintas tanto en edad como en estrato socioeconómico, se tomaron 28 estudiantes, cuyas edades oscilaban entre los 9 y los 18 años, catorce por cada institución 7 de sexo femenino y 7 de sexo masculino respectivamente. La investigación fue de tipo cualitativo, observación participante adaptada. Para la recolección de los datos se empleó el instrumento de la entrevista con las preguntas divididas para responder a las que corresponden a las segmentaciones de entrada, a las micro y macromediaciones, los diarios de campo, que nos aportaron información referente a los resultados obtenidos de las tres actividades implementadas durante el observatorio de televisión, desarrollado por los estudiantes de las dos instituciones educativas mencionadas anteriormente.

Para el análisis de los datos empleamos el protocolo de análisis de resultados de la Maestría en Comunicación Educativa de la Universidad Tecnológica de Pereira que sugiere una serie de siete pasos para la obtención y comprensión de los resultados.

Una vez analizados los resultados se logró dar respuesta a la pregunta de investigación, además se observaron las similitudes y diferencias presentadas en las producciones desarrolladas por los estudiantes participantes del observatorio de televisión de las dos instituciones educativas. El tiempo empleado para el desarrollo y análisis de los datos de la investigación fue de un año aproximadamente.

\section{Metodología}

La investigación realizada es de tipo cualitativo, la razón para escogerla fue la posibilidad de describir, analizar y explicar los datos obtenidos a partir de la observación participante. La información obtenida nos permitió acercarnos a los grupos, observar sus comportamientos, actitudes y comentarios elaborados a partir de la interacción antes y durante la realización de las distintas actividades del observatorio de televisión.

Tal como lo corrobora Douglas (citado por Deslauriers, 2004), la investigación cualitativa desarrolla su trabajo empleando datos que no son fácilmente cuantificables como los informes de las entrevistas, diarios de campo, videos, la observación participante, fotografías, historias de vida, entre otras técnicas informativas, las cuales posibilitaron el percibir las mediaciones en la vida cotidiana de los investigados.

Podemos establecer que la investigación cualitativa como parte de un sistema teórico o como paradigma nos conduce a escoger o seleccionar aquellos métodos que son aplicables y pertinentes según el contexto de la investigación, entendiendo la investigación cualitativa como:

"Campo interdisciplinar, trans-disciplinar y en muchas ocasiones contra- disciplinar. Atraviesa las humanidades, las ciencias sociales y las físicas. La investigación cualitativa es muchas cosas al mismo tiempo. Es multiparadigmática en su enfoque. Los que la practican son sensibles al valor del enfoque 
multimetódico.[..]"(Gurdián, 2007, p. 34)

Este concepto nos permitió comprender que este modelo nos acerca a aspectos más culturales, sociales, éticos y políticos, que permiten la participación y la transformación de la realidad de los contextos en donde se aplica o dirige este tipo de estudios, develando una relación diferente entre el sujeto y el objeto, en la que no es pertinente asumirla únicamente desde una perspectiva objetiva y positivista, puesto que la investigación cualitativa está dirigida por sus características a un proceso multicultural.

Para la investigación se realizó en un primer momento una etapa de diagnóstico a través de una entrevista individual en la cual se manifiestan los datos que dan como resultado, lo que se ha denominado rituales, hábitos, gustos de programación, horarios, si existe acompañamiento durante el acto televisivo, es decir aspectos propios de las micro y macro mediaciones; la entrevista se adaptó según la población, que en este caso corresponde a los estudiantes del curso 507 de la jornada tarde del Colegio Ciudadela Educativa de Bosa, conformado por niños y niñas en edades de 9 a 12 años, y los estudiantes de grado undécimo del curso 11A del colegio Gimnasio Campestre Reino Británico, jóvenes en edades entre los 14 y 16 años. Así mismo se realizó la firma del consentimiento para la recolección de la información.

Para realizar el análisis de la entrevista diagnóstica y los diarios de campo como segundo instrumento tal como se mencionó en la etapa introductoria se empleó el protocolo de la Maestría en Comunicación de la Universidad Tecnológica de
Pereira, el cual consta de siete pasos que permitieron hacer una mayor comprensión de los resultados obtenidos, de esta manera para lograr una mayor abstracción del análisis realizado a los diarios de campo, se hizo pertinente establecer las relaciones entre los datos analizados de las actividades realizadas, ya que a partir de estos podríamos reconocer cuáles eran las categorías presentes y cuál la reflexión que se podía hacer con relación a la forma en cómo los niños y jóvenes de la muestra asumen los mensajes e interpretan los contenidos que observan a través de las distintas pantallas, tal como lo muestra el siguiente ejemplo.

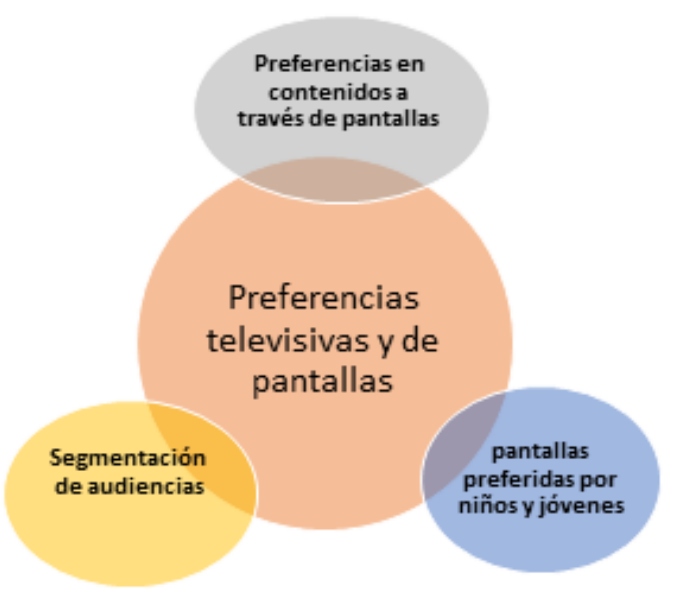

Gráfico 1. Preferencias televisivas y de pantallas

Al mencionar que están presentes la micromediaciones, las encontramos desde las televidencias de segundo orden, ya que hay una audiencia que está más allá del televisor, al interactuar con otras pantallas, pero a su vez mantienen contacto con el "referente televisivo", como lo expresa Orozco "un contacto que no es directo ni físico (audiovisual) con la imagen y el relato televisivos, sino "resonante" 
(Orozco, 2001, p. 45), es decir, que puede ser sensorial, evocado por recuerdos, que le permiten a ese televidente en cualquier momento, lugar o contexto "recontactarse" con el referente televisivo, y es precisamente lo ocurrido en la primera actividad, en la cual a través de un dibujo y haciendo uso de material de reciclaje recrearon su pantalla favorita, además de elaborar una breve explicación de las razones por las cuales era su preferida, hay una evocación de lo que les gusta de su pantalla favorita.

Un ejemplo de estas explicaciones la tenemos en el estudiante E09-F-16, quien manifiesta lo siguiente: "el televisor tiene mejor calidad HD, puedo ver películas, tiene para navegar en internet, puedo ver programas interesantes", para E06-M-16, haciendo referencia al Iphone comenta: "es fácil de llevarlo a todas partes, rápido, no le entra virus, no se bloquea, tiene acceso a internet, fácil comunicación, tiene una cámara con alta definición, puedo ver series", mientras E015-F-9, se refiere de esta manera a su pantalla favorita: "a mi me gusta el televisor, porque acá me informo de cosas, veo muñequitos, puedo escuchar música y me puedo conectar a las redes sociales", para E12-M-17: el celular es práctico, se puede escuchar música, tiene fácil acceso al internet, toma buenas fotos, fácil acceso a las redes sociales, buenas imágenes, es muy útil, buen sonido, se pueden realizar trabajos.

\section{La segmentación de audiencias y} preferencias en contenidos a través de pantallas, las pudimos explicar desde la segunda actividad del observatorio de televisión, esta se enfocó en dos momentos: individual y grupal, en el primer momento los estudiantes de las dos instituciones objeto de estudio, previamente debían ver en su casa un programa de televisión de su preferencia, usando la pantalla que ellos quisieran. Se les facilitó el modelo de un cuadro para completar con información donde se incluía el nombre del programa, tipo de lenguaje formal, informal utilizado en el contenido de este, público al que estaba dirigido, el género de los personajes, si había personajes infantiles y/o juveniles, los comerciales, la duración de estos, y el tipo de mensaje que ellos consideraban según su criterio era el transmitido por el programa.

Para explicar lo anterior podemos apoyarnos en lo manifestado por Orozco (2001, p. 34), al suponer "...que la imagen no sólo complementa sino que "habla por sí misma" y ... origina información específica al igual que el audio, el discurso, la música y los efectos incrustados en el relato televisivo, estirando la percepción, que se vuelve capaz de codificar, simultáneamente y al ritmo marcado, la multiplicidad de datos vertidos en los diferentes lenguajes."

Encontramos las segmentaciones de entrada, que tienen relación con el género y la edad, se evidencian en los contenidos vistos por los estudiantes como: Dragon Ball Z, los Simpsons, La promesa, La tusa los hombres también lloran, un show más, entre otros; en el desarrollo de esta actividad, tienen un público específico y ellos logran reconocerlo, siendo esto un factor aparentemente superficial, pero que logran identificarlo.

Para reconocer la macro-mediaciones se realizó la actividad número tres partiendo de la pregunta ¿qué puedo producir a partir del uso de las pantallas que prefiero con relación a lo que veo?, las respuestas proporcionadas por los niños y jóvenes 
respecto a la pregunta, van orientadas a la idea de crear y producir programas del género de ficción, de contenidos con sentido social, musicales para el entretenimiento y de información deportiva, entre otros, siendo los mencionados los más representativos, esto permite entender los gustos y preferencias de los estudiantes, partiendo de este ejercicio, fue posible que realizarán producciones con una visión crítica y de múltiples interpretaciones de la realidad.

\section{Resultados}

En este espacio abordaremos los principales resultados, los cuales permitirán comprender los conceptos abordados en el presente artículo

\section{Resultados obtenidos de la entrevista diagnóstica}

\section{Micromediaciones de primer orden}

La relación entre las preguntas que en el análisis de denominaron rutinas de televidencia se hace posible desde las micro-mediaciones de primer orden, tal como se puede observar en el siguiente diagrama.

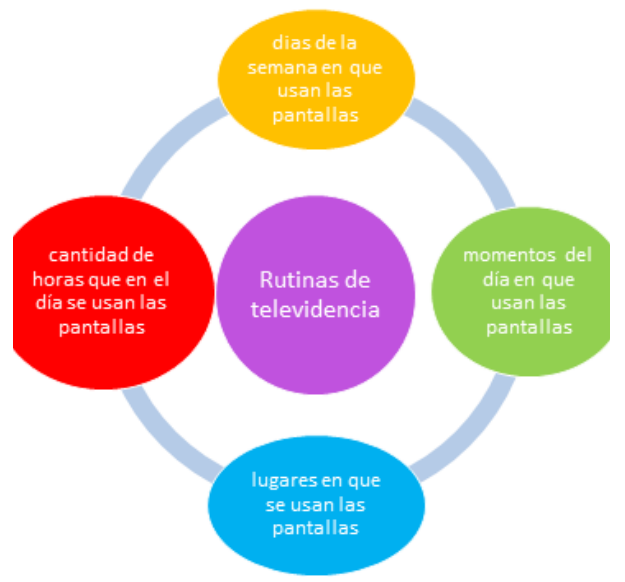

Gráfico 2. Rutinas de televidencia
La relación establecida entre lo que se puede denominar preferencias en contenidos, lo podemos ubicar en el concepto de las micromediaciones de primer orden, en estas podemos identificar que los gustos por determinados contenidos permiten reconocer que existen géneros que son de interés particular de los participantes, esto se acerca a lo que para Orozco (2001) es la segmentación propiciada por las programadoras, pero que en estas decisiones también intervienen lo educativo, lo emocional y toda la influencia de la cultura en la cual se está inmerso. Para hacer más clara esta relación hemos elaborado el siguiente diagrama, el cual permite identificar las preguntas que se relacionan con lo denominado gustos y preferencias televisivas.

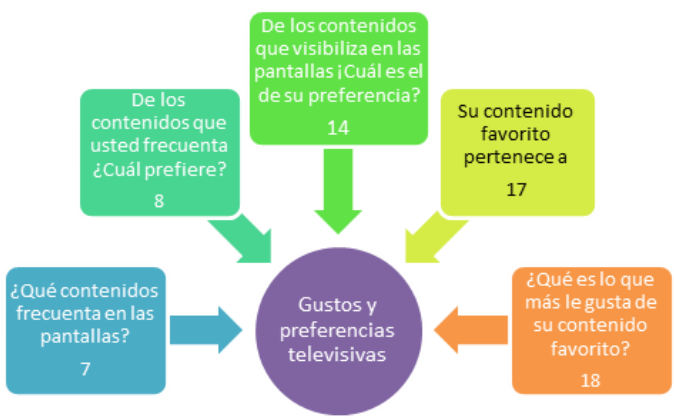

Gráfico 3 Gustos y preferencias televisivas

\section{Micromediaciones de segundo orden}

Otras de las relaciones que encontramos nos acercan a lo que hemos denominado segmentos específicos de audiencias, esta clasificación surge al reconocer que dependiendo del momento del día en que se utilizan las pantallas, es muy seguro que no se esté absolutamente solo y que en ese momento la presencia de la familia sea segura, demostrando que la mediación de la institución familiar es relevante, este es el factor común encontrado entre las 
preguntas ¿con quien comparte lo que ve en las pantallas? y ¿en qué momento del día comparte lo que ve en las pantallas?, puesto que más allá de las pantallas los sujetos-audiencia re-producen, re-crean $\mathrm{y}$ re-viven los referentes televisivos (Orozco,2000), para esta relación hemos diseñado el siguiente diagrama.

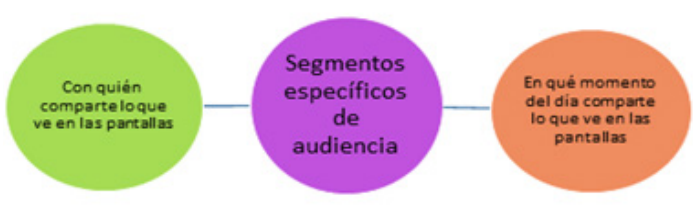

Gráfico 4 Segmentos específicos de audiencia

Para hacer referencia a las sensaciones y percepciones televisivas, hemos encontrado relación entre las preguntas que abordan las emociones, recuerdos y pensamientos que generan los contenidos y personajes favoritos, esta etapa de las sensaciones pertenece a las micromediaciones de segundo orden, ya que estas hacen referencia a las reacciones y/o evocaciones generadas a partir de lo que se observa en los contenidos televisivos, siendo así un ejemplo de ello, lo que respondieron los entrevistados, pues sus personajes y contenidos les generaba sensaciones de diversión, alegría, tranquilidad, emoción, entretenimiento, etc.

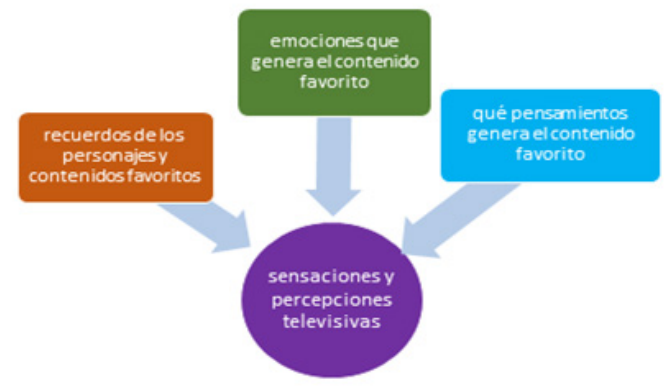

Gráfico 5 sensaciones y percepciones televisivas

\section{Macromediaciones}

Desde la categoría de las macromediaciones se puede establecer una relación entre los diferentes entornos en los cuales se desenvuelven los entrevistados y es así como se puede establecer la relación entre la forma en cómo aprendieron a usar las pantallas y con quien o quienes comparten lo que les surge cuando usan las pantallas, porque en sus respuestas, se encuentra que la familia como institución es el primer mediador en esta relación, y se puede ubicar en la categoría de las macromediaciones, ya que estas hacen referencia a las instituciones mediadoras y al papel que juegan en los diferentes contextos, como la escuela y la familia, frente a la recepción de los contenidos televisivos.

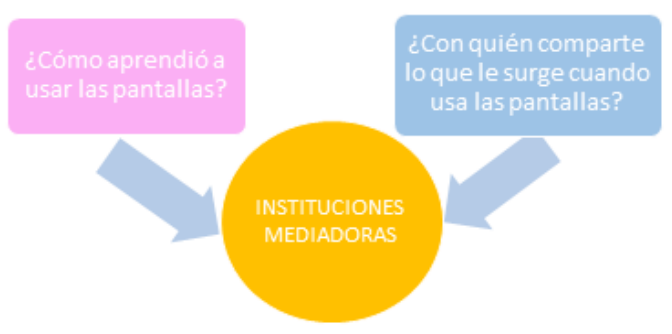

\section{Gráfico 6 Instituciones mediadoras}

En la relación establecida entre los lugares donde les gustaría encontrar pantallas, y el contexto escolar, se obtiene que varias de las propuestas ya son realidad; y las podemos encontrar en espacios como centros comerciales, servicio de transporte, incluso en los parques cuando hay eventos especiales, podemos decir que este tema se puede reconocer dentro de la categoría de las macromediaciones, puesto que estas mediaciones se dan desde el campo pedagógico y se ubican en otros contextos como lo es el "cultural-educativocomunicacional” (Orozco,2001). 


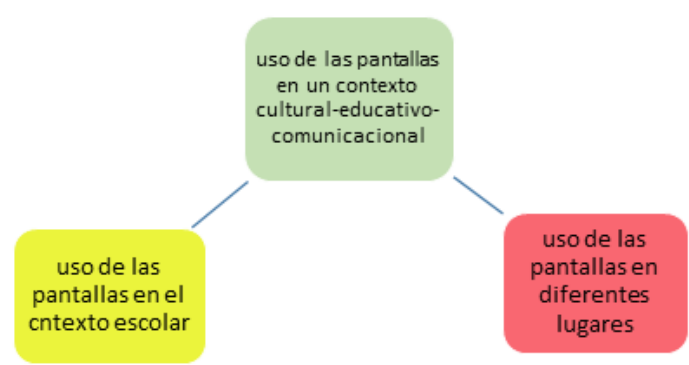

Gráfico 7 Uso de las pantallas en un contexto cultural-educativo-comunicacional

\section{Relación entremicroy macromediaciones}

Las preguntas que hacen referencia a las pantallas de mayor preferencia de los entrevistados son ¿usa pantallas?, ¿qué pantallas usa? y ¿cuál es el dispositivo en el que más consume contenidos? y se relacionan desde las mediaciones de primer orden como sujetos-audiencias con gustos muy particulares, que han propiciado también rituales y preferencias propias de cada cual y las macromediaciones muy relacionadas con las instituciones e institucionalidades, los dispositivos son facilitados por la familia como institución primaria y mediadora entre los participantes y los contenidos de su preferencia.

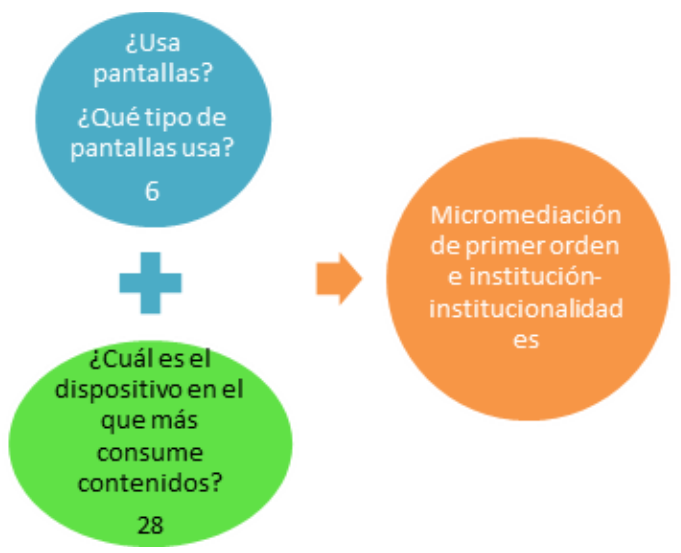

Gráfico 8 Micromediación de primer orden e institución-institucionalidades
Las preguntas ¿por qué usa las pantallas? y ¿por qué utiliza las pantallas? nos remiten a la finalidad en el uso de estas y por lo tanto tienen relación, ya que responden a subjetividades de las audiencias respecto a las sensaciones y gustos que les genera el uso de las pantallas con relación a los contenidos que ven, para mostrar esta relación elaboramos el siguiente diagrama.

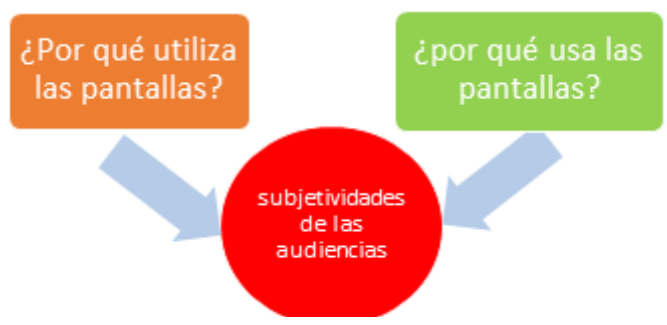

Gráfico 9 subjetividades de las audiencias

Resumen de relaciones entre los distintos temas de la entrevista diagnóstica

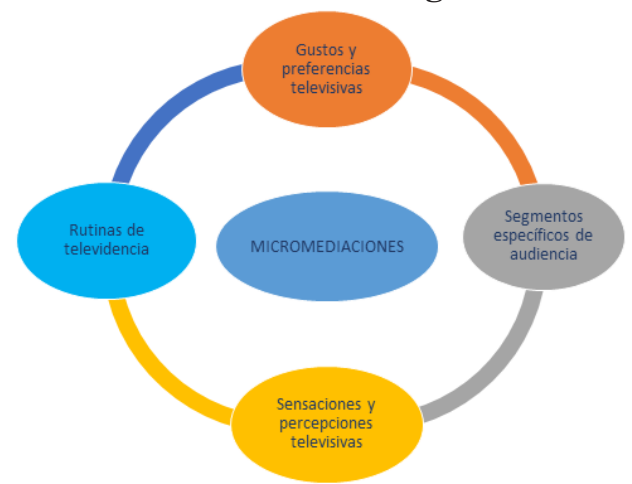

Gráfico 10 Micromediaciones

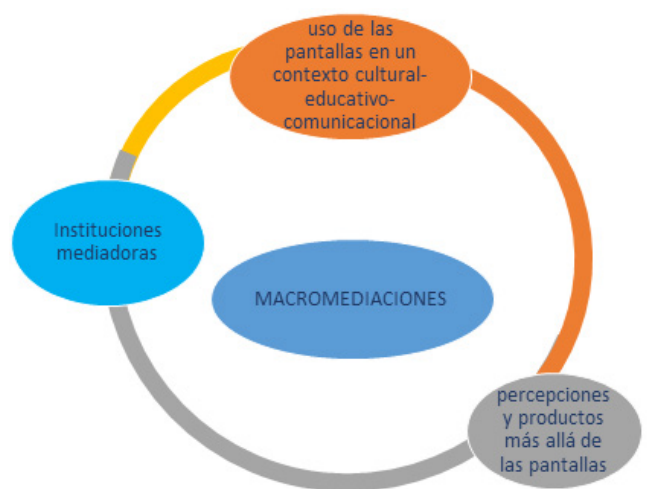

Gráfico 11 Macromediaciones 


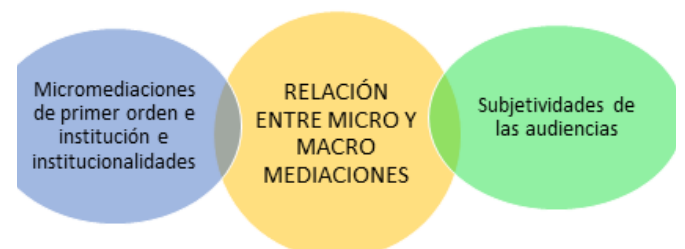

\section{Gráfico 12 Relación entre micro y macro mediaciones}

La relación entre las categorías analizadas a partir de las respuestas de la entrevista diagnóstica, proviene como lo expresa Orozco (2001) del ámbito individual inicialmente, ya que las audiencias como tales están segmentadas por sus gustos particulares y subjetividades individualizadas pero también entran en juego las macromediaciones, pues las instituciones como la familia, los amigos $y$ otros factores afectan e influyen en la manera como se aprecian los programas desde las distintas pantallas con las cuales interactúan, ya que son ellos quienes acompañan a los niños y jóvenes a la hora de ver ciertos contenidos, así mismo son aquellos con quienes interactúan y comparten ideas y percepciones de lo que ven.

Respecto a los gustos y preferencias, entran a participar todos los avances tecnológicos que se ponen en juego al servicio de las programadoras para atraer y retener a las audiencias, es decir, las tecnicidades, es aquí donde los niños y jóvenes pueden escoger una gran variedad de contenidos a partir de los géneros de su preferencia.

También encontramos otra categoría que denominamos dispersiones, hallamos que estas podían relacionarse directamente con las ya agrupadas o darnos cuenta de otras categorías pero que al hacerlas era posible denominar categorías emergentes, sin embargo al establecer estas nuevas agrupaciones encontramos que de una manera indirecta hacían parte de las categorías objeto de estudio de nuestro trabajo de investigación que eran las micro-mediaciones y las macromediaciones, pero que al relacionarlas con las demás respuestas generadas por los niños y jóvenes de la muestra no se obtuvo ninguna relación inicialmente durante el análisis de cada pregunta.

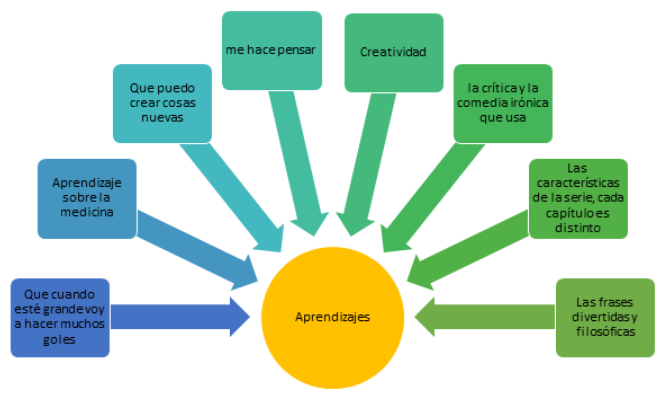

Gráfico 13 Aprendizajes

Las relaciones entre algunas dispersiones nos permitieron establecer desde las macromediaciones, la existencia de una categoría emergente la cual denominamos aprendizajes, puesto que es el resultado de la mediación de distintas fuentes que influyen también a nivel moral, cultural, social en las audiencias, las cuales en nuestro caso corresponden a una audiencia infantil y juvenil y permitió vislumbrar que esta audiencia no es pasiva frente a las emisiones, sino que son sujetos "pensantes", críticos, ante lo que están viendo y capaces de exponer sus opiniones, actitudes y valores a partir de los contenidos que ven en las pantallas.

\section{Resultados de los diarios de campo}

Para realizar el análisis de los diarios de campo como segundo instrumento se empleó el protocolo de la Maestría 
en Comunicación de la Universidad Tecnológica de Pereira, tal como se utilizó con el primer instrumento.

Las actividades realizadas para el registro de los diarios corresponde a la adaptación realizada a algunas de las actividades propuestas por Guillermo Orozco en el libro Niños, Maestros y pantallas, Observatorio de televisión en la escuela guía didáctica del alumno. (2008)

\section{Actividad Nro. 1: Por qué me gustan las pantallas}

Al revisar las explicaciones de los niños y jóvenes, como respuesta a la actividad realizada en la cual a través de un dibujo y /o haciendo uso de material de reciclaje recrearon su pantalla favorita, además realizaron una breve explicación de las razones por las cuales era su preferida, encontramos que hay preferencias por la facilidad para acceder a diferentes programas, entre los que se destacan los de ficción, los informativos y la búsqueda de información relacionada con la ampliación del conocimiento o como ayuda para realizar tareas. En cuanto a las pantallas hay gustos compartidos entre lo que se ha denominado pantallas móviles las cuales incluyen el celular, el iPhone, el iPad, la Tablet y el portátil, y las pantallas convencionales: el televisor y el computador.

Estos puntos de vista por parte de los estudiantes, podemos asociarlos con lo que plantea (Orozco, 2001, p. 49) respecto a las macromediaciones y las percepciones, en las que afirma que "la televisión no educa, pero los niños sí aprenden de ella", él a través de esta frase intenta reunir el sentir de muchos maestros en América Latina, según lo afirma una vez que emite la frase.
Retomando este referente y asociándolo con la actividad realizada, este aprendizaje trasciende al plano de las pantallas, ya que estas han cambiado la forma en que se usan y en cómo se recepcionan los contenidos que se visualizan en estas, los cuales pueden tener un fin netamente de entretenimiento y/o de aprendizaje.

\section{Actividad Nro. 2: Qué observan los niños $y$ jóvenes en los programas que ven}

El segundo ejercicio del observatorio, en el cual los jóvenes y niños de las dos instituciones Gimnasio Campestre Reino Británico y Ciudadela Educativa de Bosa, de manera individual mediante un formato emitían sus apreciaciones respecto a un programa del género de ficción de su preferencia, visualizado a través de la pantalla favorita, se pudo observar que en los programas vistos por los jóvenes de la primera institución educativa mencionada, además de la ficción mostraron interés en otros géneros como realitys e informativos, mientras que para los niños de la segunda institución la preferencia más marcada fue por la ficción, lo que nos permite afirmar que en una mayor proporción los niños y jóvenes prefieren este género, y que en su mayoria observan dichos contenidos con un fin de entretenimiento.

En esta actividad también se encuentra la segmentación de audiencias, como el identificar y clasificar el tipo de público al que va dirigido cada programa, se evidenció que los jóvenes y niños pueden reconocer el rol y la presencia de hombres, mujeres y niños y/o jóvenes en los contenidos televisivos, encontrando que el rol de mayor presencia es el masculino, hecho comprobado a partir de los datos obtenidos, por ejemplo, en un programa como "Un 
Show más", ellos identificaron en uno de los capítulos vistos, la participación de 8 personajes masculinos.

Sobre la promoción de otros programas y productos para comprar comentan en general los estudiantes que reconocen el sentido comercial de la publicidad que se oferta al interior de los distintos programas que observan, este aspecto que motiva a la compra o promoción de distintos objetos o servicios, lo podemos clasificar desde la teoría de Orozco como el "lucro mercantil", ya que su finalidad es la de promover el consumismo entre la audiencia.

Por otra parte ese amplio espectro que incluye distintos géneros como ficción, informativos, deportivos y realitys, los cuales son del gusto de los estudiantes de la muestra del observatorio; nos permite identificar que ellos escogen dentro de la oferta televisiva los que son de su agrado, sintiéndose un poco limitados, frente a dicha oferta, ya que, no son quienes hacen la programación, son otros quienes la planean y la presentan, por lo tanto no hay una real libertad, siempre se está a la merced de lo que propongan otros, tal como lo manifiesta Hall, 1982, mencionado por Orozco(2001); pero a pesar de esta sensación, en la etapa de identificar los mensajes se puede ver que esta "libertad" que sería la de elegir, les da la posibilidad de tomar posturas y expresar sus opiniones en sus contextos inmediatos, permitiéndoles reflexionar y deducir el tipo de mensaje que genera el programa que observan, de acuerdo a sus percepciones y a la influencia que han ejercido instituciones mediadoras como su familia, su religión, entre otras; aunque a algunos no les provoque ningún tipo de reflexión.

\section{Actividad Nro 3: Qué puedo producir a partir del uso de las pantallas que prefiero con relación a lo que veo}

Los niños y jóvenes iniciaron la creación de distintos productos de ficción que se caracterizaron por presentar particularidades como la semantización crítica ante los mensajes televisivos y de pantallas, es decir, se percibió el reconocimiento de los roles propios de cada género en la sociedad, asumiendo una postura reflexiva sobre lo que muestran los contenidos televisivos, lo que implica una afectación por parte de las mediaciones culturales.

Especialmente esto se observa en las producciones audiovisuales creadas, las cuales fueron tituladas FREE THE NIPPLE, TÚ NO ERES EL ÚNICO EN EL MUNDO, EL ROBO A UN BANCO y LIBERTAD DE EXPRESIÓN, el primero plantea una posición reflexiva frente al respeto y la igualdad de género, retomando una campaña existente y que ha tomado mucha fuerza en redes sociales e internet, el segundo hace una reflexión frente a los efectos de la publicidad, en cuanto al robo; los niños, manifiestan que ahora la mayoría de delincuentes son jóvenes e incluso que sus delitos quedan impunes, porque pueden hacer cualquier cosa o cometer delitos terribles y no son castigados y ellos creen que esto no debe ser así, pues consideran que sí saben lo que hacen y la cuarta producción es de tipo reflexivo, ya que invita a observar la postura que tienen los jóvenes frente a un tema como la libertad de expresión y las connotaciones que esta tiene en el contexto social.

Así mismo, estas producciones muestran cómo los niños y jóvenes hacen una lectura 
y reinterpretación de las realidades que se muestran en los distintos formatos de televisión como a través de las otras pantallas, lo que les hace posible crear nuevas narrativas, tal como lo muestran los productos elaborados, esta reflexión nos permite acercarnos al planteamiento que hace Orozco "Ser joven en tiempos de avasallamiento televisivo constituye una fuente variada, apenas explorada de mediaciones que se manifiestan de múltiples maneras, en la reconstrucción de posicionamientos..." (Orozco, 2001, p.61)

En cuanto a las canciones presentadas por las niñas, ellas manifiestan que son los temas que les gustan y las series como Violeta, tienen mucha música, las motiva porque les atrae el personaje y todo los sucesos emocionantes de la trama, cómo cantan, la forma de moverse, cómo se visten, cómo bailan, les encantan todas las canciones, se podría decir, que sienten simpatía y se identifican con la protagonista, siguiendo la trama del programa con avidez, tanto es así, que las niñas contaron que si promocionan conciertos donde se presentan las protagonistas, ellas van.

Desde Orozco (2001), podríamos asegurar que esta producción es reflejo o consecuencia de lo que él denomina "identidades de moda", las cuales son impuestas por la televisión y que también tiene la participación de los demás medios, que colaboran en el posicionamiento dentro de las audiencias.

También observamos tomando como referencia la canción de Reggaeton 6 AM, que es muy posible que sólo tengan en cuenta el ritmo de la canción y el baile que es muy "pegajoso", pero no se han detenido a analizar el contenido del tema, la letra de la canción, esto nos sugiere indagar acerca de la comprensión de la letra. Si sólo la cantan porque gusta y/o están entendiendo los mensajes y sus significaciones, que para los adultos son más evidentes, pero esto sería tema de otro estudio partiendo de cuestionamientos como el si "¿Los estudiantes comprenden el mensaje de las canciones de Reggaeton?"

\section{Discusión de resultados}

Para realizar la discusión de los resultados nos ubicamos nuevamente en los conceptos de audiencia, recepción y mediación, para entender los términos de micro y macro mediaciones, ya que es a partir de estos que se generó el trabajo realizado. Estos términos los abordamos desde la teoría de Orozco, quien en su texto Televisión, audiencias y educación los define como:
Audiencias es aquí asumida como un conjunto segmentado a partir de sus interacciones mediáticas de sujetos sociales, activos e interactivos, que no dejan de ser lo que son mientras entablan alguna relación siempre situada con el referente mediático, sea ésta directa, indirecta o diferida (Orozco, 2001.p. 45)

Para el observatorio de televisión, a nuestro grupo de trabajo lo denominamos audiencia infantil y juvenil, ubicándolos dentro de las características mencionadas por el autor, por otro lado tenemos el concepto de recepción que hace referencia a la...

$[\ldots]$ interacción
mediada desde diversas
fuentes y contextualizada


material, cognitiva y emocionalmente, que se despliega a lo largo de un proceso complejo situado en varios escenarios $\mathrm{y}$ que incluye estrategias y negociaciones de los sujetos con el referente mediático de la que resultan apropiaciones variadas que van desde la mera reproducción hasta la resistencia y la contestación. (Orozco, 2001, p. 23)

En este caso la recepción la tomamos como ese proceso de interacción que las audiencias hacen con el referente televisivo y las pantallas y que de esta se puede generar una reacción a partir de lo que observan.

Ubicar esta recepción de audiencias en un plano de interpretación, de orientación y de "otorgamiento de un sentido", dentro de un contexto o escenario solo se logra desde la mediación, que para Orozco es entendida como "un proceso estructurante que configura y orienta la interacción de las audiencias y cuyo resultado es el otorgamiento de sentido por parte de éstas a los referentes mediáticos con los que interactúan" (Orozco, 2001, p. 23)

Continuando en el plano de las mediaciones y para comprender mejor este proceso nos acercamos a la teoría de Martín Barbero, de la que se entiende que son importantes en la medida en que se consideran instrumentos de "transformación cultural", convirtiéndose en espacios de re-significación que las audiencias o receptores hacen de la cultura de modo que "en la medida en que la tecnología materializó cambios que desde la vida social daban sentido a nuevas relaciones y nuevos usos, estamos situando los medios en el ámbito de las mediaciones,[...]" (Barbero,1987:154).

Así mismo relacionamos este concepto con lo que definió Fagerjord en (Scolari, 2009) a partir de esas nuevas relaciones y significaciones y es el concepto de las "convergencias retóricas", las cuales se entienden como " diferentes estilos y sistemas de signos que combinan en complejos textos y significaciones, selecciones a cargo del lector y procesos semióticos(Fagerjord, 2003.p207)[...] el uso del término retórica [...] dejaría la puerta abierta a una posible taxonomía de las diferentes figuras de la convergencia entre lenguajes y medios" (Scolari, 2009, p. 52).

Al referirnos a las mediaciones y convergencias, podemos establecer la relación televisión - audiencia y es precisamente el análisis de esta relación la que nos brinda la posibilidad de hablar de micro y macromediaciones, las cuales son evidentes en la investigación desde la entrevista diagnóstica realizada en el observatorio de televisión, ya que en esta se pudo identificar algunas de sus características y diferentes aspectos como el de las segmentaciones de entrada, las cuales se evidenciaron al preguntar a los participantes por su edad, el estrato socioeconómico, y con quién viven, reconociendo en este punto que la familia ejerce una función mediadora frente a la observación de los distintos programas que fueron surgiendo a medida que se iba revisando las respuestas a las preguntas del formato adaptado de la maestría de la UTP. 
Encontramos que el $77 \%$ de los estudiantes de la muestra comparten con su familia lo que ven en las pantallas, en ese sentir de ser audiencia y tal como lo manifiesta Orozco (2001, p. 24), “...ser audiencia también modifica el vínculo fundamental entre los sujetos sociales con su entorno y con los acontecimientos y fuentes tradicionales de información: barrio, amigos, familia, compañeros de trabajo o de juego[...]", esto lo podemos ejemplificar a partir de respuestas como las de la pregunta con quién comparte lo que ve en las pantallas en la que uno de los jóvenes E02P19 -M15 respondió; con mis hermanos o padres, otro E08P19 -M- 16; con mi familia y amigos, otro E27 P19 - M - 11; con mi papá y mi mamá y mis dos hermanos, estas respuestas nos acercan a esa interacción de la audiencia con su entorno.

Desde este sentido, es pertinente recordar que las micromediaciones se manifiestan en el momento en que los estudiantes como audiencia toman la decisión de ubicarse frente a la pantalla del televisor o cualquier pantalla de su preferencia y comienzan a buscar ese referente televisivo que los atrae, aquí entran en juego los gustos, percepciones, hasta las rutinas y los distintos rituales que dan origen a los hábitos, seguramente a la misma hora, día tras día se ubican frente a la pantalla buscando ese programa que se destaca entre los otros ofertados por la programadora.

Como parte de las micromediaciones también encontramos que las respuestas de los estudiantes a la pregunta cuál es la pantalla favorita, revelan que es el televisor la pantalla que adquiere mayor relevancia, seguida muy de cerca por el celular y el computador, aunque aquí sí influye la edad, refiriéndonos a quienes poseen un celular, en su mayoría son los jóvenes y no los niños; sin embargo los menores también son atraídos por otras pantallas como la Tablet y el celular, sin perder el interés por el tv, tal como lo podemos evidenciar en las respuestas generadas por los niños y jóvenes de la muestra: E02P06-M-15 expresa que tiene gusto por diferentes pantallas y hace referencia al celular, televisor, portátil, iPad, EO10P06-M-16 responde que sus preferencias incluyen celular, computador, iPad, televisor, EO24P06-M-1 dice T.V., computador, E026P06-M-11, responde computador, Tablet, televisor.

Las macromediaciones, las hallamos en algunas preguntas como por ejemplo las referentes a la forma como aprendieron los estudiantes a utilizar las distintas pantallas, en la entrevista diagnóstica, identificamos que un 50\% encontró respaldo en su grupo familiar, mientras las respuestas obtenidas para la segunda actividad, la cual se denominó lo que observan los estudiantes en los programas que ven y que fueron registradas en los diarios de campo, hacen referencia a las televidencias de segundo orden, pues estas nos permiten identificar cómo los niños y jóvenes de la muestra comparten información a partir de los contenidos que ven.

En el momento en que los estudiantes realizaron un producto a partir de lo que ven en las pantallas en la primera parte que era de carácter individual, los estudiantes debían dar respuesta al siguiente interrogante ¿Que puede producir con lo que ve en las pantallas? las respuestas muestran que ellos se dan la posibilidad de retomar sus recuerdos, hacer evocaciones, de hacer uso de su imaginación para recrear, y reproducir las apropiaciones que de los distintos referentes televisivos 
ellos hacen. Por ejemplo E015-F-9 nos manifiesta lo siguiente. "Produciría un programa de muñequitos, porque nació mi hermanita y me gustaría inventarla para que ella, se divierta y se ría"; E03-F-16, dice "crearía campañas de concientización, frente a casos como la trata de personas, previniendo al aumento de estos".

Continuando con las macromediaciones, tenemos la que para Orozco (2001, p. 46) es una identidad de "moda", y son propiciadas $\mathrm{y} / \mathrm{o}$ impuestas tanto por la televisión como por los otros medios, es el caso de las canciones presentadas por las estudiantes del Colegio Ciudadela Educativa de Bosa; 6 AM, tema del género Reggaeton, se escucha cantar a los estudiantes y se puede observar que el video es emitido con bastante frecuencia en las distintas pantallas, de la canción tomamos el siguiente estribillo:

“...y ya son las seis de la
mañana
y todavía no recuerdo nada
ni siquiera conozco tu cara
pero amaneciste aquí en mi
cama
pero qué clase e' rumba pa,
pa, pa
la que yo tuve anoche
que, que, que, no recuerdo lo
que sucedió,..."

Así mismo encontramos que la canción "Todo por Amor" de la serie Violeta, atrae a las niñas y jovencitas con su trama y melodías; y la manera como expresan lo que perciben los jóvenes del Gimnasio a través de sus producciones audiovisuales, por ejemplo en la que se hace una crítica al consumo, la campaña por la igualdad de género FREE THE NIPPLE y para entender este proceso de producción nos acercamos al concepto de hipermediación que propone Scolari, el cual plantea que "al hablar de hipermediación no nos referimos tanto a un producto o un medio sino a procesos de intercambio, producción y consumo simbólico que se desarrollan en un entorno caracterizado por una gran cantidad de sujetos, medios y lenguajes interconectados tecnológicamente de manera reticular entre sí."(Scolari, 2008, pág.113).

Partiendo del concepto de hipermediación, podemos establecer cierta similitud con otros como el de convergencias, sobre este aspecto Roberto Igarza, mencionado por Germán Muñoz(2010, pág. 10) nos dice que los jóvenes nativos digitales que nacieron entre el televisor y el computador efectúan una (...) circulación fluida de información y entretenimiento entre medios: usan todo tipo de intercambios comunicativos alternativamente, en dispositivos polifuncionales en los que se integran textos, imágenes y audios(...). Esta interconexión entre las distintas pantallas permite accesibilidad a contenidos de interés de manera inmediata y posibilita que exista un intercambio de información y a la vez creación de mensajes, de nuevos puntos de vista, de nuevas ideas.

Tomando como base este concepto se puede entender que el proceso de producción, comprensión y asimilación de los contenidos visualizados en la televisión $\mathrm{y}$ en las diferentes pantallas, pueden ser generadores de nuevas narrativas, nuevos discursos, como resultado de la interacción en un contexto y a partir de la recepción de diferentes factores discursivos, tecnológicos, comerciales, académicos, etc., los cuales modelan una visión de 
mundo que posibilita la socialización e interacción de sus propios puntos de vista, opiniones y posiciones críticas, tal como se evidencia en las respuestas dadas por los estudiantes de la muestra en las actividades realizadas.

Por ejemplo E02-M-15 sobre los Simpson respondió que este programa hace una crítica a la sociedad (religión, política, consumismo, economía, pobreza), E03-F-16, respecto a La promesa, expresa que este invita a concientizar a la población frente a estos casos (trata de personas), E10-M- 16, sobre Dragon Ball $\mathrm{Z}$ dice que se puede reflexionar sobre la paz, ya que muestra violencia y agresividad, E015-F-9, sobre La Rosa de Guadalupe, manifiesta que hay que respetar a las personas, así sean sirvientes, choferes o incluso callejeros, así que a respetarlos, no seas indiferente, esta afirmación con relación a las respuestas de los jóvenes en la segunda actividad del observatorio la podemos argumentar a partir de lo que expresa Guillermo Orozco

[...] los televidentes ni son pasivos, ni acríticos, sino todo lo contrario.[...]

La nueva figura del prosumidor, que es a la vez consumidor y productor de contenidos mediáticos, parece que guiará las nuevas formas de interacción entre medios audiencia. Aunque el primer paso es el de formarnos como receptores y consumidores críticos frente a las pantallas, pues no se puede producir algo cuando no se entiende cómo funcionan medios como la televisión e internet (Orozco, 2010, pág. 31)
Desde esta posición es que podemos afirmar que los niños y jóvenes al convertirse en receptores críticos de los contenidos vistos a través de las diferentes pantallas, tienen la posibilidad de ser productores de nuevos contenidos y narrativas, las cuales muestran la posición que han tomado como audiencia.

\section{Conclusiones.}

Con la realización de las distintas actividades propuestas durante el desarrollo del observatorio de televisión, buscamos dar respuesta a la pregunta objeto del proyecto y se llegaron a las siguientes conclusiones.

Encontramos que el televisor, el computador, el celular y la Tablet son las pantallas preferidas por los niños y jóvenes de la muestra y a partir de estas, muchos realizan interconexiones, expandiendo el acceso a otras fuentes de información o entretenimiento, confirmando lo que autores como Roberto Igarza llaman "convergencias" y para Scolari son "hipermediaciones", por ejemplo manifiesta E12-M -17: el celular es práctico, se puede escuchar música, tiene fácil acceso al internet, toma buenas fotos, fácil acceso a las redes sociales, buenas imágenes, es muy útil, buen sonido, se pueden realizar trabajos, mientras para E09-F- 16: el televisor tiene mejor calidad HD, puedo ver películas, tiene para navegar en Internet, puedo ver programas interesantes.

Se identificó que los contenidos de ficción a los que con mayor frecuencia acceden los estudiantes de la muestra son clasificados en dibujos animados en la población infantil y series en la juvenil, seguido de 
un variado grupo de contenidos como películas, programas musicales, y saliendo un poco del plano de ficción encontramos los documentales, realitys, el uso y acceso a las redes sociales y el uso de la internet para el entretenimiento y consulta de información general.

Podemos afirmar que las micromediaciones se encuentran presentes en todas las actividades desarrolladas en el observatorio, nos dimos cuenta que desde lo individual cada estudiante tiene sus apreciaciones, valores, referentes familiares, puesto que su entorno es muy particular, pues en este han crecido, han ido desarrollando sus ideas, puntos de vista; como respaldo a lo anterior Orozco (2010) hace referencia a que aunque sean respuestas individuales está implícita la influencia que ejerce su familia, su cultura y todos los factores que los afecten como las amistades y sus comentarios, las charlas en el colegio exponiendo sus opiniones sobre lo observado.

Respecto a las macromediaciones, identificamos que la familia como primera institución que influye de muchas maneras en las videncias que realizan tanto jóvenes como niños, no realiza un acompañamiento significativo durante la observación de los distintos tipos de programas que ven los estudiantes, pues en la recepción de contenidos de ficción, incluyen programas que pertenecen según su clasificación a la franja de adultos y se les permite el libre acceso a estos, lo cual podemos comprobar en la entrevista diagnóstica, donde respondieron que a la hora de acceder a algún contenido lo hacen solos la mayor parte del tiempo y aparentemente no tienen restricción.
A partir de la realización del observatorio de televisión podemos afirmar que los estudiantes no son tan pasivos ante las pantallas como podríamos pensar, sino que tienen una capacidad reflexiva frente a los mensajes que transmiten los contenidos televisivos de ficción y que son capaces de realizar o generar productos que muestren dichas reflexiones, es así como lo describimos en cada uno de los diarios de campo, especialmente en el correspondiente a la última actividad en la que los jóvenes tuvieron la oportunidad de crear un producto representado y haciendo uso de algún recurso digital, audiovisual, la forma de interpretación y propuestas respecto a lo que ven en los contenidos televisivos y de pantallas.

Dichas producciones se enfocaron en la recreación de realidades de ficción, en la reproducción de situaciones que son de su agrado, en la manifestación de puntos de vista y reflexiones, algunas de las producciones fueron la campaña FREE THE NIPPLE, la crítica a la sociedad de consumo(tú no eres el único en el mundo), el apoyo a la libertad de expresión, temas recurrentes en hechos noticiosos y de ficción como lo es un robo o asalto y mostrados con mensajes implícitos en los contenidos que ven los niños y jóvenes como en los programas y videos musicales.

Con las producciones como resultado del observatorio de televisión, podemos establecer que no existe una relación directa entre televisión y escuela, pero que sí es posible generar aprendizajes, lo cual nos invita a los docentes a replantear el discurso y vincular el discurso televisivo como estrategia dentro de los procesos de enseñanza - aprendizaje, para esto podemos apoyarnos en lo que 
plantea Guillermo Orozco "Al volver conscientes los aprendizajes mediáticos de sus alumnos, el maestro no solo tiene la capacidad de incorporar a los medios al proceso educativo, sino de hacerlo a favor de una educación integral que permita a los alumnos tener armas frente al "bombardeo" informativo del que son presas a diario"(Orozco, 2010, P. 21)

\section{Referencias}

Barker, C. (1952) frases, recuperado de http:// www.proverbia.net/citasautor.asp?autor $=1289$

Barbero, J., (1987). De los medios a las mediaciones, comunicación, cultura $\mathrm{y}$ hegemonía. Editorial Gustavo Gili, $2^{\mathrm{a}}$ edición, Barcelona p. 154

Charles, C., M., Orozco, G., G., (1991) El niño como televidente no nace, se hace En Hacia una lectura crítica de los medios. pp. $33-48$, Editorial Trillas, México.

Gurdián, F. A. (2007). El paradigma cualitativo en la investigación Socio-Educativa. Recuperado de

http://web.ua.es/en/ice/documentos/recursos/ materiales/el-paradigma-cualitativo-en-lainvestigacion-socio-educativa.pdf

Muñoz, G. G. ¿De los "Nuevos medios" a las "hipermediaciones"?, Revista Latinoamericana de Ciencias Sociales, Niñez y Juventud. Manizales, Doctorado en Ciencias Sociales, Niñez y Juventud del Centro de Estudios Avanzado en Niñez y Juventud de la Universidad de Manizales y el Cinde, vol. 8, núm. 1 (separata), enero-junio de 2010, pp. 9-16

Orozco, G., (2001). Audiencias, Televidencias y Mediaciones: un reconocimiento preliminar en Televisión, audiencias y educación. Pp $39-$ 62. Grupo Editorial Norma Buenos Aires.
Orozco G., MIGUES, F., D., (Primera edición, 2010). Niños, maestros y pantallas Observatorios de televisión en la escuela: Guía didáctica para el maestro preliminar en Televisión, audiencias y educación. Pp 19 - 37. Grupo Editorial Norma Buenos Aires.

Scolari, C., (2008). Hipermediaciones, elementos para una teoría de la comunicación digital interactiva. (Primera Edición). España, Editorial GEDISA, S.A. p. 113. Recuperado dehttp://comunicacion3unlz.com.ar/wpcontent/uploads/2014/07/HipermediacionesCarlos-Scolari-Cap1.pdf

Scolari, C., (2009). Alrededor de la(s) convergencia (s)1 Conversaciones teóricas divergencias conceptuales y transformaciones en el ecosistema de medios En Revista Signo y pensamiento 54, Volumen (XXVIII), 4455. Recuperado de http://revistas.javeriana. edu.co/index.php/signoypensamiento/article/ viewFile/4527/3489 